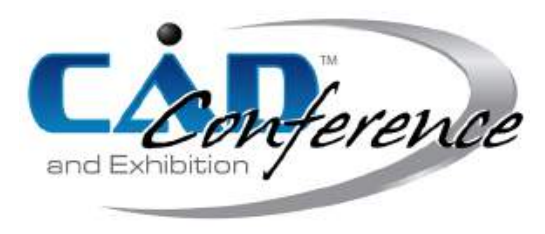

Title:

\title{
Topology Control and Simplification of Reality-based Meshes for Finite Element Analysis
}

Authors:

Marco Rossoni, marco.rossoni@polimi.it, Politecnico di Milano

Sara Gonizzi Barsanti, sara.gonizzi@polimi.it, Politecnico di Milano

Giorgio Colombo, giorgio.colombo@polimi.it, Politecnico di Milano

Gabriele Guidi, gabriele.guidi@polimi.it, Politecnico di Milano

Keywords:

Reverse Engineering, Topology, Retopology, Finite Element Analysis

DOI: $10.14733 /$ cadconfP.2019.392-396

\section{Introduction:}

Reverse Engineering has been exploited for several application, e.g measuring and inspection, customfit design, cultural heritage. The result of this process is a $3 \mathrm{D}$ representation of the targeted object, usually encoded in an unstructured triangulated surface fashion (e.g. an STL file). Going beyond the mere visualization purpose and focusing on engineering products, prediction of mechanical performances is often required, especially for items critical from a structural perspective. Finite Method Analysis (FEA) is a de-facto standard to evaluate mechanical performances, both in academia and industry, but the meshes derived from reverse engineering techniques are not suitable for direct use in FEA solver. In those cases, a proper degree of accuracy, precision and reliability throughout the whole pipeline have to be guaranteed, from the acquisition of the geometry to the analysis of the final result. Regardless the reverse engineering technology chosen, the standard pipeline requires the acquisition of the chosen object and the post process of the $3 \mathrm{D}$ model obtained in order to have a closed $3 \mathrm{D}$ mesh. The most important and thorny phase is the one related to the topological check of the $3 \mathrm{D}$ mesh and its simplification to obtain a proper model, suitable to be then translated in a computational grid for FEA. The problem is not new in literature [4] but researches have been focused more on large items (e.g. buildings). Firstly, the order of magnitude of the relevant feature is different: talking about architectural items, small features, i.e. less than $1 \mathrm{~mm}$, are negligible. Secondly, similar researches conducted so far don't evaluate FEA results systematically.

This paper aims at comparing different methodologies in order to prepare computational mesh of geometries derived from reverse engineering technologies. A benchmark case, i.e. a structural-steel parallelepiped, has been chosen in order to have complete control over the variables involved in the process (both during the reverse engineering and the FEA). The test object has been acquired with a laser scanner and post processed in order to fix artifacts. Once the mesh is closed and error-free, two different methodology for simplification have been used: a triangular simplification and retopology. The acquired geometry, before and after the simplifications, has been compared with the reference model (i.e. a 3D geometry created using CAD geometric primitive): mean and standard deviation between the baseline model and the acquired geometries has been tracked. Finally, a tensile test has been simulated making use of a FEA software and the results have been compared with the theoretical solution. 


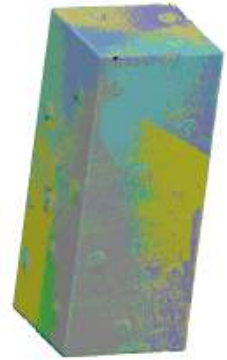

(a)

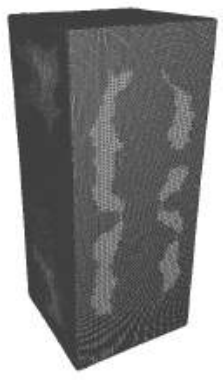

(b)

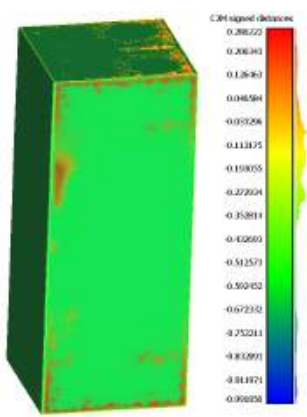

(c)

Fig. 1: (a) Aligned scans in Polyworks (b) Mesh generated from the point cloud acquired by the laser scanner (c) Comparison between the generated mesh and the nominal parallelepiped 50x50x120 mm

Reverse Engineering, Topology and quality control:

Reverse engineering aims at taking something as a software, an object or a device apart to analyze its functionality and with the intention of constructing a new object or device starting form the initial one [9]. Topology can be defined as the study of qualitative properties of certain objects or how elements of a set relate spatially to each other. A good topology for a 3D model means avoiding self-intersecting polygons, holes, duplicated vertices or faces, non-manifold faces. A mesh is a manifold if each edge is incident to only one or two faces. For the quality check of the achieved $3 \mathrm{D}$ results, there are different approaches and methods. Most of the applications using 3D models require such models to be geometrically and visually accurate and free from noise, out layers and missing data or holes. Not only such errors make the models unusable for documentation or reproduction but also create unpleasant visual experience. As seen, topology is referred to the study of geometrical properties and spatial relations between the polygons of a mesh. Triangular meshes are defined by a series of triangles which barycenter describes a linear surface representation. A mesh can be described by a set of vertices and a set of triangles connecting them even if is more efficient to define the triangular mesh with the edges of the polygons [3].

\section{Survey:}

The test object for this project is a structural steel parallelepiped of 50x50x120mm of dimension. It was decided to use this object because of its simple geometry and on the other hand, sharp edges that can lead to problems during the survey and topological errors in the final $3 \mathrm{D}$ mesh. The survey has been carried out through laser scanning techniques with a Minolta Vivid910 triangulation laser scanner equipped with a tele-lens that, considering the dimension of the object and the distance of scanning, gave an uncertainty of $43 \mu \mathrm{m}$. The parallelepiped was surveyed with 17 scans, moving the object in front of the scanner in order to have the entire surface covered (Fig1a). InnovMetric Polyworks has been used for the alignment and, at the end of this phase, the mean standard deviation was $0.05 \mathrm{~mm}$, close to the uncertainty of the instrument. Even if this software is able to repair and generate meshes, only the point cloud has been saved: since it is a proprietary software, it does not let the user full control over the algorithm used to generate the mesh. Starting from the point cloud, the final mesh (188314 faces and 94159 vertices, Fig.1b) has been generated using Screened Surface Poisson Reconstruction algorithm [7], already implemented in Meshlab. The quality check of the topology was done in Meshlab, applying several filters to clean the data: (i) Remove duplicate faces/vertex; (ii) Remove faces from Non Manifold edges; (iii) Remove unreferenced vertex; (iv) Remove zero area Faces; and (vi) Select and cancel non Manifold Edges/vertices. This step is mandatory in order to obtain a closed superficial mesh to be converted into 


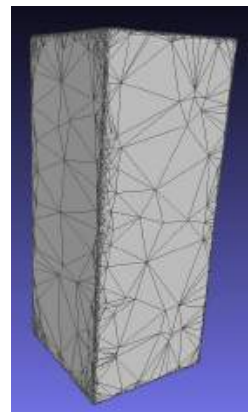

(a)

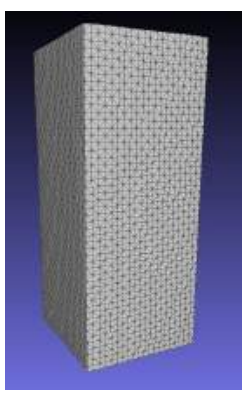

(b)

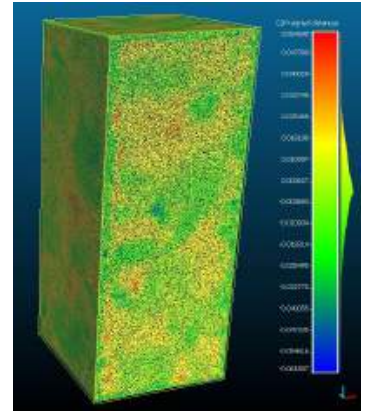

(c)

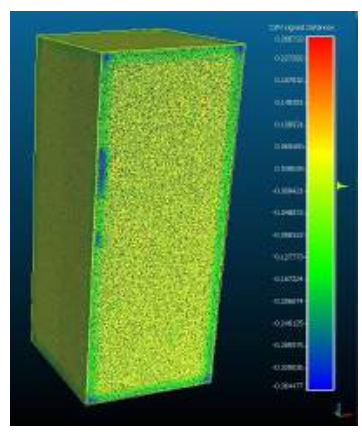

(d)

Fig. 2: (a) Triangular simplification, 9.6K polygons; (b) Retopology, 9.6K polygons;(c) Comparison between the high resolution mesh derived from the acquisition phase and the triangular simplified one; (d) Comparison between the high resolution mesh derived from the acquisition phase and the retopologized one

a closed volume, suitable for FEA. In order to verify the acquisition, a comparison between the generated mesh and the nominal parallelepiped 50x50x120 mm has been performed. As shown in Fig.1c, the mean error is $0.21 \mathrm{~mm}$ with a standard deviation of $0.15 \mathrm{~mm}$. As expected, the greatest errors are along the edges. This problem, namely laser scattering [8], shows up due to the sharp edges and it is related to the laser technology itself. The model in Fig.1b will be the reference model for the following simplification and retopology phase.

Simplification of the meshes and creation of volumetric models for FEA:

The simplification process is to obtain a surface $M^{1}$ as similar as possible to the initial high resolution mesh $M$. There are different approaches, the majority of which involves the degradation of the mesh to reduce the number of polygons [11],[6]: (i) vertex decimation [10] deriving from the Delaunay Pyramid method first proposed in [5]; (ii) energy function optimization algorithm; (iii) agglomeration of vertices (or Vertex clustering); (iv) region Merging/Face Clustering.

Quad-based topology consists of rows and columns, a simple topology and an outcome easily to be subdivided, while a model with triangle-based topology can product sharp angles that can affect the design of a mesh. The quadrangular method samples the original mesh at a spatial resolution lower than the original with a degree of accuracy higher than a triangular mesh, because it preserves the global geometry of the original mesh, re defining its topological structure [1]. Retopology [2] is the creation of a new topology for a 3D model and it is obtained by laying down a low-polygon mesh over top of the high density model and it starts a new polygonal organization that follows the main geometrical feature of the main 3D object. For this project, Polyworks and the open source Instant Meshes software were used to produce a simplified triangular and quadrangular mesh, imposing the number of polygons on $9 \mathrm{~K}$. Instant Meshes permits to correct the superficial distribution of the elements, in order to have a better organization of the geometry. Both 3D simplified models have been checked again in their topology in Meshlab.

The comparison among the high-res mesh, the triangular simplification and the retopologized one was performed with CloudCompare and gave a standard deviation of $0.01 \mathrm{~mm}$ and $0.05 \mathrm{~mm}$ respectively (Fig.2c, 2d). As expected, the triangular simplified mesh gave better results because in the retopologized process a smoothing is added to the new mesh. Anyway, both standard deviation results are acceptable, while in the order of the uncertainty of the scanner used. 


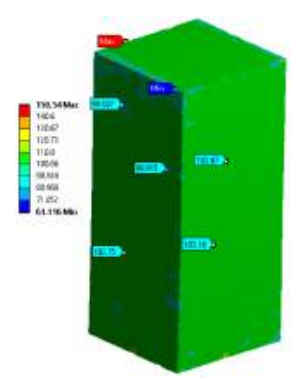

(a)

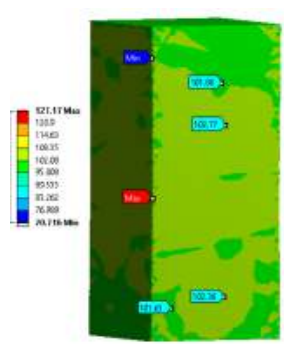

(b)

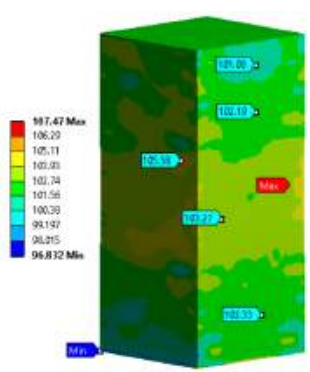

(c)

Fig. 3: Normal stress contour expressed in MPa of (a) As-Is mesh; (b) Triangular simplification, 9.6K polygons; (c) Retopology, 9.6K polygons;

Finite Element Analysis Settings and Results:

A tensile test has been simulated making use of a FEA software (i.e. Ansys Mechanical R19.2). This simple test allows the results to be compared with a theoretical solution. The parallelepiped is supposed to be fixed and loaded with $100 \mathrm{kN}$ (traction), at the bottom and top side respectively. Given that the cross-sectional area is $2500 \mathrm{~mm}^{2}$, the tensile stress is $100 \mathrm{MPa}$ constant along the whole piece. This stress is the reference value. It has been preferred to set three different boundary conditions in order to avoid singularities due to over-constrained elements at the base. The mesh has been generated as follow: (i) import the unstructured mesh (i.e. stl file); (ii) surface meshing with a Patch Conforming algorithm, in order to respect edges and vertices for a given part; (iii) the Advancing Front Tetra mesher method (bottom-up) has been used to fill the internal volume with tetrahedrons. A global seed of 2 $\mathrm{mm}$ and a curvature/proximity based refinement allowed till minimum edge length of $0.5 \mathrm{~mm}$ are the size parameter for the tetrahedrons. The chosen element order is quadratic. Since the input meshes are different among the as-scanned (Fig.1b), the simplified (Fig.2a) and the retopologized one (Fig.2b), the resulting computational meshes will be different as well: the distinction is only on the number of elements given that the other parameters has been fixed.

The FEA results for the three models are summarized in Fig.3. As expected, all the three models present a non-uniform and non-constant distribution of the stress. It can be ascribed to the noise introduced by the laser scanner and, to the fact that the cross-section is not perfectly $2500 \mathrm{~mm}^{2}$. The AS-IS model was hard to mesh due to its high resolution mesh. Even with a so simple geometry, the FEA software lasts long time in order to complete the mesh without gaining any advantage over the other two models. However, the retopologized model presents a stress distribution that is less sparse than the other two. Furthermore, due to a more uniform mesh, it has been handled easier and more efficiently by the FEA software. Finally, the range of normal stress of the retopologized model is more close to the true value $(100 \mathrm{MPa})$ : this is a sign that the initial mesh, in this case, leads to better FEA results. Fig3a and Fig.3b present also pronounced peaks of stress: local singularities are responsible for those. This phenomenon is less evident in Fig.3c.

\section{Conclusion:}

This work investigates how meshes originated from laser scanner affects the generation of computational grid for FEA and the related results. A benchmark specimen (i.e. 50x50x120 mm steel parallelepiped) has been used in order to provide quantitative outcomes and keep under full control the whole pipeline. As-is scanned mesh reconstruction leads to high resolution models: high number of triangles can lead to unsuitable and heavy mesh, difficult to be converted in a computational grid. The simplification process 
is a mandatory step in order to have a mesh suitable to be converted in a volumetric model for FEA. Even if retopology adds a smoothing on the simplified mesh, the reorganization of the quadrangular elements on the surface avoids sharp, non-manifold edges and permits a stronger simplification of the model maintaining the accuracy of the high-resolution one.

Findings of our research can be summarized as follow: (i) laser scanner still lacks on detecting sharp edges (essential for most engineering product), consequently they are the greatest source of geometrical error; (ii) simplification and retopology don't introduce excessive geometric approximations (mean error are about $10 \mu \mathrm{m}$ and $4 \mu \mathrm{m}$ respectively) but considerable advantages in the FEA mesh generation; and (iii) geometric inaccuracies (i.e. irregular surface that should be planar face instead and sharp edges) is reflected in inaccurate FEA results, mainly due to inaccurate application of boundary conditions and loads.

As future work, the benchmark will be surveyed with two different devices: a structured light device that is supposed to provide more accurate results especially regarding sharp edges and a desktop 3D scanner that uses an array of lasers to scan objects at high resolution. In this way, the artifacts discovered on the final high resolution mesh are supposed to be avoided. This results will be compared also with a photogrammetic mesh. The idea is to analyze pro and cons of each technique, to find the best to be used for the survey of manufacturing objects, then exposed to FEA.

\section{References:}

[1] Attene, M.; Campen, M.; Kobbelt, L.: Polygon mesh repairing: an application perspective. ACM Computing Surveys, 45(2), 2013, 1-33. https://doi.org/10.1145/2431211.2431214

[2] Bommes, D.; Zimmer, H.; Kobbelt, L.; Mixed-integer quadrangulation. ACM Transactions on Graphics, 28(3), 2009, 1-10. https://dx.doi.org/10.1145/1531326.1531383

[3] Botsch, M.; Kobbelt, L.; Alliez, P.; Lévy, B.: Polygonal Mesh Processing, AK Peters/CRC Press, New York, 2010.

[4] Castellazzi, G.; D'Altri, A.; Bitelli, G.; Selvaggi, I.; Lambertini, A.: From laser scanning to finite element analysis of complex buildings by using a semi-automatic procedure. Sensors, 15(8), 2015, 18360-18380. https://doi.org/10.3390/s150818360.

[5] De Floriani, L.: A pyramidal data structure for triangle-based surface description. IEEE Computer Graphics and Applications, 9(2), 1989, 67-78. https://doi.org/10.1109/38.19053

[6] Franc, M.: Methods for Polygonal Mesh Simplification. PhD, thesis, University of Bohemia, Pilsen, 2002

[7] Kazhdan, M.; Hoppe, H.: Screened poisson surface reconstruction. ACM Transactions on Graphics, 32(3), 2013. https://doi.org/10.1145/2487228.2487237

[8] He, Y.; Liang, B.; Yang, J.; Li, S.; He, J.: An iterative closest points algorithm for registration of $3 \mathrm{D}$ laser scanner point clouds with geometric features. Sensors, 17(8), 2017. https://doi.org/10.3390/s17081862

[9] Raja, V.; Fernandes, K.J.: Reverse Engineering: An Industrial Perspective. Springer, London, 2008. https://doi.org/10.1007/978-1-84628-856-2

[10] Soucy, M.; Laurendeau, D.: A general surface approach to the integration of a set of rangeviews. IEEE Transactions on Pattern Analysis and Machine Intelligence, 17(4), 1995, 344-358. https://doi.org/10.1109/34.385982

[11] Taime, A.; Saaidi, A.; Satori, K.: Comparative study of mesh simplification algorithms. Proceedings of the Mediterranean Conference on Information \& Communication Technologies, 380, Springer, Cham, 2016. https://doi.org/10.1007/978-3-319-30301-7 